\title{
Role of MRI in evaluation of oral cavity cancers from central India
}

\author{
Valecha $\mathbf{J}^{1}{ }^{1}$, Ojha S. $^{2}$, Tripathi P. ${ }^{3}$ \\ ${ }^{1}$ Dr. Jyoti Valecha, Associate Professor, ${ }^{2}$ Dr. Sandeep Ojha, Associate Professor, ${ }^{3}$ Dr. Purva Tripathi1, DNB, Senior \\ Resident, all authors are affiliated with Chirayu Medical College, Bhopal, MP, India.
}

Corresponding Author: Dr. Jyoti Valecha, Associate Professor, Department of Radiology, Chirayu Medical College and Hospital, Bhopal. Email: drjyotivalecha@yahoo.co.in

\begin{abstract}
Introduction: Squamous cell carcinoma of oral cavity ranks in the top three types of all cancer in the India. The diagnosisof it is basically clinical and bioptic, preoperative imaging is crucial for tumor staging that helps to decide appropriate therapeutic strategy and indicate prognosis. Objectives: To evaluate the diagnostic accuracy of Magnetic resonance imaging (MRI) in staging of oral cavity cancers and to correlate the results with clinical and pathological data. Methodology: This was a prospective study of 60 patients of oral cavity squamous cell carcinomas (SCC) where MRI was performed preoperatively to determine the tumor staging, tumor thickness, infiltration of surrounding structures and bone invasion. These findings were correlated with final pathological staging. Results: Accurate correlation of $\mathrm{T}$ stages (TNM system) was obtained in 51 out of 60 cases on MRI. One lesion was classified as T2 on MRI but was found to be T4 on histopathology as MRI failed to detect subtle infiltration of the cortical bone. The accuracy of clinical data in T stage evaluation was $62 \%$ (K- 0.470), however the MRI accuracy found to be $82 \%$ (k 0.740$)$. The sensitivity, specificity and accuracy of MRI in the detection of mandibular involvement were $94.1 \%, 60 \%$ and $81.5 \%$, respectively. Conclusion: MRI plays an important role in the evaluation of oral cavity carcinomas. MRI accurately demonstrates the tumor size, depth of invasion, bone marrowinvolvement, perineural spreadandlymphnodemetastasiswhichare essentialfor treatmentplanning.
\end{abstract}

Key words: MRI, Oral Squamous Cell Carcinoma, Clinical Staging, Pathological staging

\section{Introduction}

Oral cavity squamous cell carcinoma (SCC) is the most common cancer in Indian subcontinent, the age adjusted incidence in India being 20 per 100,000 population [1]. Increase incidence in Indian population is because of the habits like tobacco, betel nutchewing and alcohol intake [2]. Oral cavity carcinomasare classified into following sub sites- Buccal mucosa, alveoluswith gingiva, hard palate, retro molar trigone, tongue and floor of mouth [3].

Clinical examination often under estimates the extent of tumor, the in filtration and invasion of deeper structures may not be appreciated and majority of metastatic lymphnodeless than $10 \mathrm{~mm}$ being interpreted as regular cervical lymphnode status on clinical examination. Hence a preoperative imagingis essential for tumor staging and appropriate treatment plan. MR imaging

Manuscript received: $28^{\text {th }}$ May 2018

Reviewed: $6^{\text {th }}$ June 2018

Author Corrected: $14^{\text {th }}$ June 2018

Accepted for Publication: $18^{\text {th }}$ June 2018 provides superior soft tissue contrast, direct multiplanar formats without ionisingradiation and better depicts the detailed anatomy of the oralcavity compared with CT. It accurately delineates the borders and extent of tumor in filtration determining the depth of invasion, involvement of critical structures, including bone and the internal carotid artery and the perineural spread the seare theim portant factors in treatment planning [4].

This study evaluated the diagnostic accuracy of MRI in the T stage of TNM classification and its loco- regional spread.

Correlation with clinical and pathological staging was done and final pathological staging was considered as gold standard. In the subgroups of the tumor close to the mandible, the relationship between tumor and mandible was also determined. 


\section{Material and Method}

Design - Prospective Study

Source of Data- This was a prospective study on 60 patients conducted in a Tertiary care unit named Chirayu Medical College and hospital, Bhopal over a period of Two years between January 2016 and January 2018.

Inclusion Criteria- All the biopsy proven patients referred for MRI staging who undergo surgeryas the first treatment modality.

\section{Exclusion Criteria}

- Patients with metallic implants,

- Patients staging more than T4A in whom non surgical treatment is the first line of treatment.

- Previously treated or recurrent oral cavity tumors were excluded from the study.

Procedure- Before doing MRI study, the clinical history and detailed local examination of oral cavity was recorded. All dentures and partial mobile dental prostheses were removed prior to clinical examination. Examination of the mucosa of the cheek, vestibule of the mouth, gums, hard and soft palate, floor of the mouth, retro molar trigone, tongue and tonsils was done to look for any ulcer, growth or any bulge. Evaluation of tongue movements were looked for, palpation of the tongue and floor of the mouth was done to detect small lesion infiltration and superficial extension. Tumor data including site, subsite, size, infiltration of the adjacent structures were recorded.

MRI examination were performed with a 1.5 Tesla. GE machine, using a head and neck phased array coil. Axial and coronal spin-echo T1-weighted (TR, 500-600 ms; TE, 7-10 ms); axial and sagittal fast spin-echo T2-weighted (TR, 3000$4000 \mathrm{~ms}$; TE, 90-100ms); coronal short tau inversion recovery (STIR) (inversion time, $150 \mathrm{~ms}$ ); and post contrast axial, coronal, and sagittal T1-weighted sequences. The sequences are acquired at 4-mm thickness with 1-mm intersection gap. The matrix used is 256 x256, NEX 2 and FOV $240 \mathrm{~mm}$. Echo-planar diffusion weighted imaging is performed with b values of 0 and $1000 \mathrm{sec} / \mathrm{mm} 2$.

Table-1: AJCC 7TH Edition 2010 (TNM Classification).

\begin{tabular}{|c|c|}
\hline Tumor Size & \\
\hline $\mathrm{TX}$ & Primary tumor cannot be assessed \\
\hline T0 & No evidence of primary tumor \\
\hline Tis & Carcinoma in situ \\
\hline T1 & Tumor $2 \mathrm{~cm}$ or less in greatest dimension \\
\hline $\mathrm{T} 2$ & Tumor more than $2 \mathrm{~cm}$ but not more than $4 \mathrm{~cm}$ in greatest dimension \\
\hline T3 & Tumor more than $4 \mathrm{~cm}$ in greatest dimension \\
\hline $\mathrm{T} 4 \mathrm{a}$ & $\begin{array}{l}\text { (oral cavity ) Moderately advanced local disease - Invades through cortical bone, into } \\
\text { deep (extrinsic) muscles of tongue, maxillary sinus, or skin of face. }\end{array}$ \\
\hline $\mathrm{T} 4 \mathrm{~b}$ & $\begin{array}{l}\text { Very advanced local disease - Invades masticator space, pterygoid plates, or skull base } \\
\text { or encases internal cortical artery node. }\end{array}$ \\
\hline \multicolumn{2}{|l|}{ Nodes } \\
\hline $\mathrm{NX}$ & Regional lymph nodes cannot be assessed \\
\hline No & No regional lymph node metastasis \\
\hline N1 & Metastasis in a single ipsilateral lymph node, $\leq 3 \mathrm{~cm}$ in greatest dimension \\
\hline $\mathrm{N} 2 \mathrm{a}$ & Metastasis in single ipsilateral lymph node, $>3 \mathrm{~cm}$ but $\leq 6 \mathrm{~cm}$ in greatest dimension \\
\hline $\mathrm{N} 2 \mathrm{~b}$ & Metastases in multiple ipsilateral lymph nodes, none $>6 \mathrm{~cm}$ in greatest dimension \\
\hline $\mathrm{N} 2 \mathrm{c}$ & Metastases in bilateral or contralateral lymph nodes, none $>6 \mathrm{~cm}$ in greatest dimension \\
\hline N3 & Metastasis in a lymph node $>6 \mathrm{~cm}$ in greatest dimension \\
\hline \multicolumn{2}{|l|}{ Metastasis } \\
\hline M0 & No distant metastasis \\
\hline M1 & Distant metastasis \\
\hline
\end{tabular}




\section{Original Research Article}

The parameters which were assessed on MRI study - size \& tumor thickness, soft tissue extent, perineural spread and marrow invasion and then given an MR T stage of the lesions. Contrast-enhanced T1W images helped to assess marrow invasion, perineural spread, soft tissue extent, tumor thickness and best demonstrate necrosis in nodes. Also it was used to measure tumor thickness for tongue and buccal sub-sites which was a latero-medialand not a cranio-caudal dimension for the vast majority of tumors that arise from the lateral border. STIR images had high sensitivity for visualizing nodes but with reduced specificity. Diffusion weighted images were of added value, particularly in sub centimeter non necrotic metastatic nodes. Noncontrast T1 weighted sequences demonstrate cortical erosion of bone. To establish the mandible involvement, loss ofhypointense line of the cortex and replacement of high signal intensity of medullary bone on nonenhanced SE T1sequencesin axial or coronalplane.

The staging of oral cavity squamous cell carcinoma was done based on American joined committee on cancer (AJCC) TNM system. The 7th edition of AJCC (2010) staging for oral SCC is provided in Table 1 [5].

All the patients below stage T4 a according to the TNM staging were included in study and hence were submitted to surgery and histopathological examination. Radiological findings were compared with final histopathological staging.

Statistical Methods- The data collected were tabulated for TNM staging of the oral cavity SCC. The collected data was analyzed by using Cohen's k coefficient of agreement and binary classification i.e. - the sensitivity, specificity, accuracy, positive predictive value (PPV) and negative predictive value (NPV).

Landis and Koch proposed categories for judging values less than 0.0 was poor, 0.00 to 0.20 was light, 0.21 to 0.40 was fair, 0.41 to 0.60 was moderate, 0.61 to 0.80 was substantial and 0.81 to 1.00 was perfect. In the evaluation of the $\mathrm{T}$ stage the accuracy of clinical and MRI stage was assessed.

In the evaluation of mandible, the sensitivity, specificity, accuracy, positive predictive value (PPV) and negative predictive value (NPV) of the MRI was assessed.

\section{Results}

The most commonly involved site of primary was buccal mucosa (Figure 1) accounting for 53.45\%, followed by tongue (33\%) (Figure 2) and retromolartrig one and alveolar ridge in $13.55 \%$ cases (Table 2). In T stage evaluation of oral cavity tumors, a positive correlation was found between the MR and pathological staging. The accuracy of clinical data in T stage evaluation was $61 \%$, K.470, however the MRI accuracy found to be $82 \%$ K.740 (Table 5). MR recorded up staging in 8 cases of the 60 patients with primary tumors and sub staging in 1 case (Table- 4). The lesion was mistakenly considered T2 stage at MR but was found to be T4 on histopathology, because of lack of detection of very small infiltration of mandibular corticalbone on MRI.

Table-2: Incidence of Individual Cancer

\begin{tabular}{|c|c|}
\hline Site & Percentage \\
\hline Buccal mucosa & $53.45 \%$ \\
\hline Tongue & $33 \%$ \\
\hline Alveolus and RMT & $13.55 \%$ \\
\hline Total & $\mathbf{1 0 0} \%$ \\
\hline
\end{tabular}

Table-3: Correlation between Clinical and Pathological T stage

\begin{tabular}{|c|c|c|c|c|c|c|}
\hline \multirow{2}{*}{ Clinical data } & \multicolumn{3}{|c|}{ Pathological Data } & \multicolumn{2}{c|}{ Total } \\
\cline { 2 - 7 } & & T1 & T2 & T3 & T4 & \multicolumn{1}{c|}{} \\
\cline { 2 - 7 } & T1 & 4 & & & 1 & 14 \\
\hline & T2 & 4 & 10 & & & 15 \\
\cline { 2 - 7 } & T3 & & 10 & 5 & & $\mathbf{2 6}$ \\
\cline { 2 - 7 } & T4 & 1 & 5 & 2 & 18 & $\mathbf{6 0}$ \\
\hline
\end{tabular}


Table-4: Correlation between MRI and pathological data T stage

\begin{tabular}{|c|c|c|c|c|c|c|}
\hline \multirow[b]{3}{*}{ MRI data } & & \multicolumn{4}{|c|}{ Pathological Data } & \multirow[t]{2}{*}{ Total } \\
\hline & & T1 & T2 & T3 & T4 & \\
\hline & T1 & 6 & & & & 6 \\
\hline & T2 & 2 & 21 & & 1 & 24 \\
\hline & T3 & & 3 & 5 & & 8 \\
\hline & T4 & 1 & 2 & & 19 & 22 \\
\hline Total & & 9 & 26 & 5 & 20 & 60 \\
\hline
\end{tabular}

Table-5: The kappa value for clinical and MRI data

\begin{tabular}{|c|c|c|}
\hline \multirow{2}{*}{ Clinical Data } & Kappa & Accuracy \\
\cline { 2 - 3 } & $\mathbf{0 . 4 7 0}$ & $\mathbf{6 2 \%}$ \\
\hline MRI Data & $\mathbf{0 . 7 4 0}$ & $\mathbf{8 2 \%}$ \\
\hline
\end{tabular}

Regarding bone infiltration three false positive cases and onefalse negative case were found in the present study on MRI imaging. Considering the three cases of false positive bone infiltration, having gingiva (two cases) andretro molar trigone tumors (one case), the supposed cortical infiltration of the mandible was not con- firmed at histopathological examination, so pathological staging resulted as pT1 (one case) and pT2 (two cases), respectively, The sensitivity, the specificity and the accuracy of MRI in the detection mandibular involvement were $94.1 \%, 60 \%$ and $81.5 \%$ respectively, while the positive and negative predictive values were $80 \%$ and $85.7 \%$ respectively.

The cervical lymph nodes metastasis was found in 34 cases out of 60 cases, level I b lymph node was the commonest node to be involved. The tumors with depth of invasion greater than $9 \mathrm{~mm}$ shown cervical nodal metastasis at one or other level in $70 \%$ of patients. However nodal metastasis detected in $12 \%$ of patients in whom tumor thicknesswas less than $3 \mathrm{~mm}$.

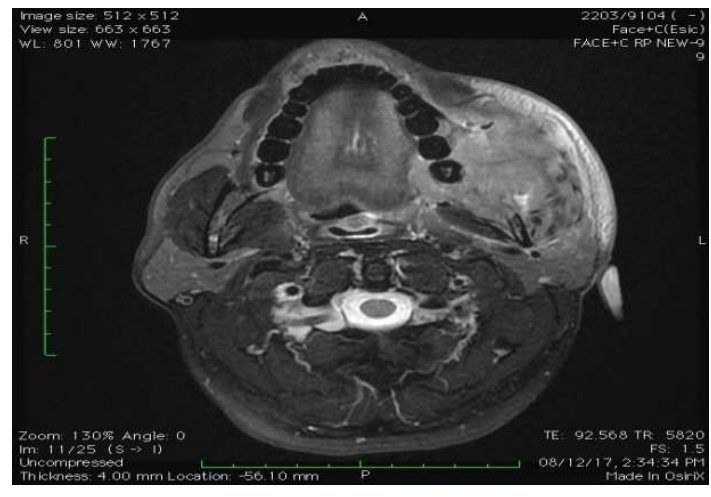

Figure-1: Axial contrast enhanced MRI showsbulky soft tissuemassin left gingiva- buccalregion extending into RMT and eroding loweralveolusand angleoflefthemi mandible.

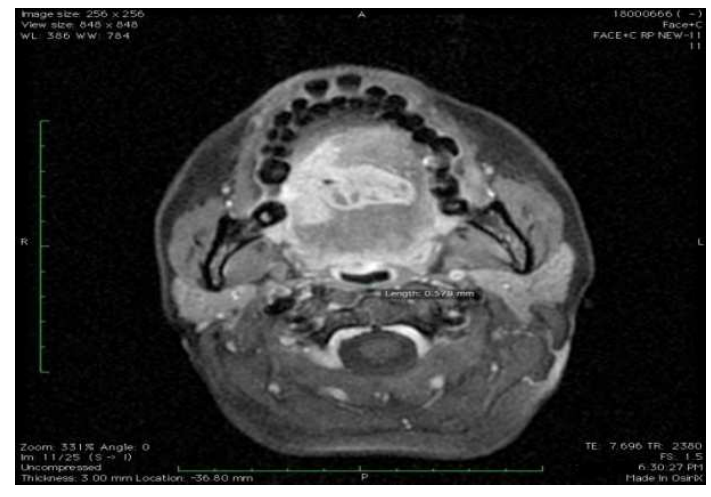

Figure- 2: Axial post contrast T1 fat sat image shows a large enhancing mass lesion in right lateral border of tongue with contralateral extension.

\section{Discussion}

For appropriate treatment planning of the oral cavity carcinomas an adequate assessment of tumor staging is essential. Multiple studies validate increased tumor size is associated with reduced overall survival, hence the size is important $\mathrm{T}$ factor on the AJCC chart and it influence both the 5-year survival rates and rates of positive cervical lymphadenopathy [6]. Evaluation of involvement of surrounding structures, apart from tumorsize, is also important in these tumors to decide $\mathrm{T}$ stage. Owing to excellent soft tissue contrast and multi planer views, MRI better delineates tumor size and thickness, local extension into surrounding structures, perineural invasion and bone infiltration [7].

In this study the, the accuracy of MRI seems to be far higher than physical examination. The diagnostic accuracy of MRIT staging compared with histo pathological staging was $82 \%$, while clinical $\mathrm{T}$ staging showed a diagnostic accuracy 


\section{Original Research Article}

of $62 \%$. Similar accuracy of clinical and MRI was also seen in a study done by Vidri A et al, predicting T stage accuracy of both clinical and MRI examination to be $62 \%$ and $82 \%$, respectively [8]. There is moderate agreement between the clinical and MRI T staging. A general tendency to over stage the tumor was more evident in cases of physical examination than MRI Similar observation was seen in the study performed by Paiboon JJ et al, which also show that mis- staging by clinical examination was higher compare to MRI [9]. The substantial agreement (K value 0.740$)$ for the MRI T staging compared with histopathological staging was noted. Zeng et al. also conducted similar studies and founded that MRI showed good performance in displaying tumor borders, depth of invasion andlocal extension which is consistent with present study [10]. The high incidence of T4 lesions in this study is related more to the facility of infiltration of surrounding structures like extension to the extrinsic muscles, encasement of neurovascular bundle, invasion of the FOM and base tongue, (upstaging disease to T4a) (according to TNM criteria [Table 1] than to the considerable sizes of the tumors. Involvement of adjacent structures, as opposed to the $\mathrm{T}$ stage, is more important in these tumors (figure 3). The accuracy of MR with regard to the evaluation of the relationship between the tumorsand surrounding structures washigh, more than $90 \%$ in this study. This has important implications in the treatment of disease in planning surgery as previously discussed [11]. Apart from tumor size, MRI alsodetermines the depth of invasion preoperatively (figure 4), which is one of the primary predictors of the nodal metastasis Okura, et alevaluated the features of primary tongue SCC on MR imaging to predict cervical nodal metastases and found that a tumor thickness of $>9.7 \mathrm{~mm}$ was a significant predictor for nodal metastases and proposed that elective neck dissection could be performed in such cases [12]. The similar findings, $70 \%$ of patients with a radiological tumor thickness of more than $8.5 \mathrm{~mm}$ showed evidence of cervical lymph node metastasis in this study.

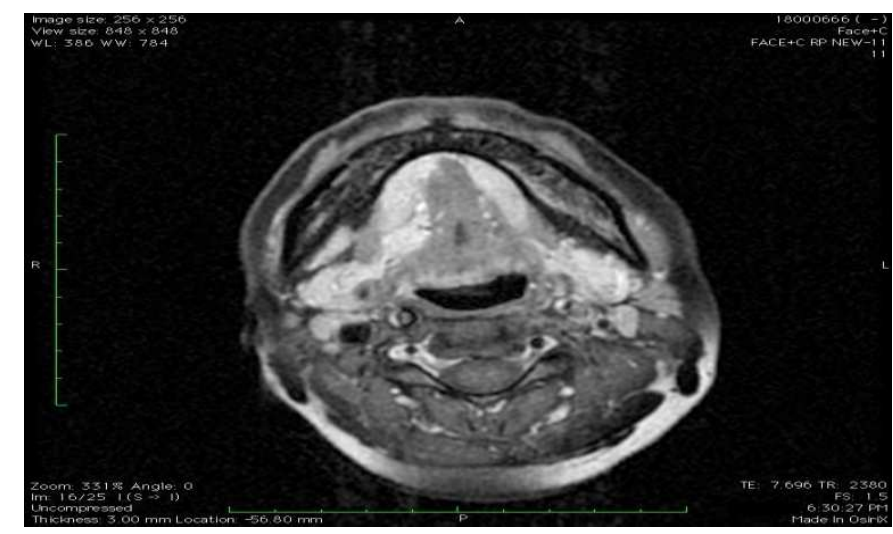

Figure-3: Axial contrast enhanced MRI shows tongue tumor extendsintosublingual space with neurovascular bundle invasion.

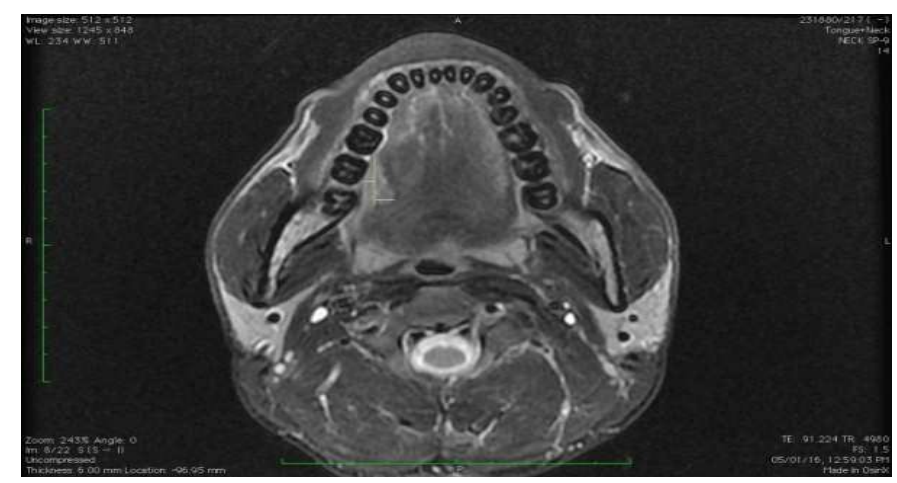

Figure-4: Demonstrates calculation of depth of invasion, the reference green line represents line drawn between the two tumor mucosal junctions and whitelineson either side of it represents perpendicular measurements at thepoint of maximal tumor projection which are added to get tumor thickness.

For assessment of bone marrow invasion MRI is superior, it can detect involvement of bone marrow, even in the absence of discontinuity of the cortical bone. It has high sensitivity $95 \%$, an accuracy of $87 \%$ and a lower specificity (75\%) in establishing bone marrow infiltration. These results agree with those reported in other studies where the specificity was $71 \%$, the accuracy $77 \%$ and the sensitivity $100 \%$ [13]. The diagnosis of the infiltration of the cortical bone of the 
Original Research Article

mandible is made on the basis of the loss of the low-signal line of the cortical bone. MRI has low sensitivity $66 \%$ and accuracy of 93 for the evaluation of the cortical bone of the mandible. In this study one lesion was mistakenly considered T2 at MR because of lack of evidence of very small in filtration of mandibular cortical bone. Hence the overall sensitivity, the specificity and the accuracy of MRI in the detection of mandibular involvement were $94.1 \%, 60 \%$ and $81.5 \%$ respectively and the positive and negative predictive values were $80 \%$ and $85.7 \%$, respectively.

Presence of cervical lymph nodes metastasis is one of the most important prognostic factors with an associated reduction of $50 \%$ in patient survival. MRI is also used to assess the extent of lymphadenopathy. The commonly using parameter of the nodal involvement on conventional MR imaging techniques are size, shape and centrally necrosis. Presence of central necrosis is the most reliable sign of malignancy [14]. These parameters are not adequate to discriminate benign from malignant nodes. In recent studies diffusion weighted imaginghas become the main sequence is used to assess the presence of occult metastatic lymph nodes [15]. The mean ADC value for malignantand benign node was $0.8 \times 10-3$ and $1.2 \times 10-3 \mathrm{~mm} 2 / \mathrm{sec}$ respectively (figure 5 ).

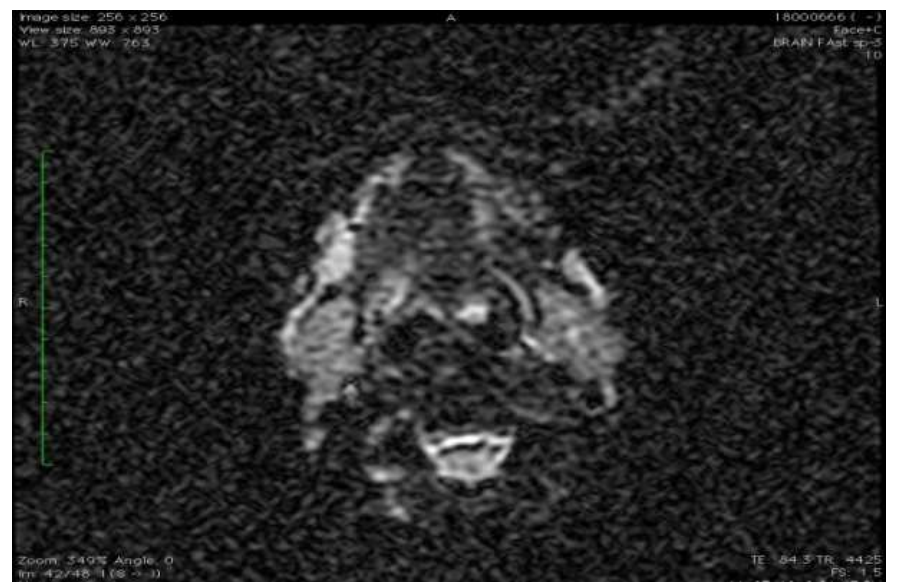

Figure-5: Diffusion weighted images on $b=1000 \mathrm{sec} / \mathrm{mm} 2$ shows hyperintensity in non- necrotic level $1 \mathrm{~b}$ lymph nodes bilaterally.

Cervical lymph node metastasis was found in 34 cases out of 60 cases in our study, with the most commonly involved lymph node level I B.

Limitations- For small superficial lesions additional techniques are needed to better assess the lesion. Artifacts seen mostly in olderage groups due to motion and dental filing magnetic susceptibility causing filed distortion. Such artifacts some times leads to false interpretation of images

\section{Conclusion}

In conclusion, MR showed a high correlation with histopathology in the evaluation of $\mathrm{T}$ stage and relationship with surrounding structures and bone marrow invasion. The depth of invasion inoral cancers can beassessed and measures satisfactory on MRI which helps in predicting cervical lymph nodal metastasis. Diffusion weighted imaging could be considered an important supportive tool to differentiate between benign and malignant cervical lymph nodes and valuable for predicting occult cervical nodal metastasis. Hence MRI is the preferred modality of choice for staging of oral cavity malignancy which helps the clinician for planning of treatment.

Funding: Nil, Conflict of interest: None

Permission of IRB: Yes

\section{References}

1. Sankaranarayanan R, Masuyer E, Swaminathan R, et al. Head and neck cancer: a global perspective on epidemiology and prognosis. Anticancer Res. 1998 Nov- Dec; 18 (6B): 4779-86.

2. Pintos J, Black MJ, Sadeghi N, Ghadirian P, Zeitouni AG, Raphael P, et al. Human papilloma virus infection and oral cancer: a case-control study in Montreal Canada. Oral Oncology. 2008; 44 (3): 24250 .

3. Lenz M, Hermans R. Imaging of the oropharynx and oral cavity. Part II: Pathology. Eur Radiol. 1996;6(4): 536-49. 


\section{Original Research Article}

4. Mari Hagiwara, Annette Nasbaum, Brian L Schmidt. Assessment of oral cavity carcinoma. Magnetic resonance imaging clinics august 2012:20 (3):473- 494

5. NCCN Guidelines Version 1. 2012, Cancer of the Oral Cavity, National Comprehensive Cancer Care Clinical Practice Guidelines in Oncology (NCCN Guidelines). NCCN.org.

6. Tytor M, Olofsson J. Prognostic factors in oral cavity carcinomas. Acta Otolaryngol Suppl. 1992;492:75-8.

7. Hagiwara M, Nusbaum A, Schmidt BL. MR assessment of oral cavity carcinomas. DOI:10.1016/j. mric. 2012.05.003

8. Vidiri A, Ruscito P, Pichi B, Pellini R, Covello R, Sperduti I, Di Giovanni S, Spriano G, Crecco M. Oral and base of the tongue tumors. Correlation between clinical, MRI and pathological staging of primary tumor. Journal of Experimental and Clinical Cancer Research. 2007 Dec 1;26(4):575.

9. Moore MA, Yoo KY, Tuncer M. What is the future for the Asian Pacific Journal of Cancer Prevention (and Control) and the Asian Pacific Organization for Cancer Prevention (and Control)? Asian Pac J Cancer Prev. 2009 Jan-Mar;10(1):1-2.

10. Zeng H, Liang $\mathrm{CH}$, Zhou ZG, Zheng JH, Zeng QX. Study of preoperative MRI staging of tongue carcinoma in relation to pathological findings. Di 1 junyi da xuexuebao $=$ Academic journal of the first medical college of PLA. 2003 Aug;23(8):841-3.

11. Rodney R. Million, Nicholas J. Cassisi, ilius, J. Blipincott Co., A Multi disciplinary Approach. Journal of the sciences and specialties of the head and neck. Volume 7, Issue 3 January/ February 1985 pages 260.

12. Okura M, Iida S, Aikawa T, Adachi T, Yoshimura N,Yamada T, Kogo M. Tumor thickness and paralingual distance of coronal MR imaging predicts cervical node metastases in oral tongue carcinoma. American Journal of Neuroradiology. 2008 Jan 1;29(1): 45-50.

13. Chung TS, Yousem DM, Seigerman HM, Schlakman BN, Weinstein GS, Hayden RE. MR of mandibular invasion in patients with oral and oropharyngeal malignant neoplasms. American journal of neuroradiology. 1994 Nov 1;15 (10): 1949-55.

14. Kumaran PS, Thangaswamy SV, Navaneetham A. The need for early detection of neck nodal metastasis in squamous cell carcinoma of oral cavity. DOI:10. 4103 /0975-7406.100300

15. de Bondt RB, Hoeberigs MC, Nelemans PJ, et al. Diagnostic accuracy and additional value of diffusionweighted imaging for discrimination of malignant cervi cal lymph nodes in head and neck squamous cell carcinoma. doi:10.1007/s00234-008-0487-2. Epub 2009 Jan 10 .

\section{How to cite this article?}

Valecha J, Ojha S, Tripathi P. Role of MRI in evaluation of oral cavity cancers from central India. Int J Med Res Rev 2018;6(05):285-291.doi:10.17511/ijmrr. 2018.i05.08. 